Pérez Fernández, P.D., Fernández Rodríguez, M., García Vega, E. y Guerra Mora, P. (2021). Acoso escolar y y autoconcepto en personas trans. Revista de Investigación Educativa, 39(1), 189-208.

DOI: http://dx.doi.org/10.6018/rie.420001

\title{
Acoso escolar y autoconcepto en personas trans
}

\section{Bullying and Self-concept in trans people}

\author{
Pablo Daniel Pérez Fernández*, María Fernández Rodríguez**, Elena García Vega* y Patricia \\ Guerra Mora*** \\ *Facultad de Psicología, Universidad de Oviedo. \\ **Unidad de Tratamiento de Identidad de Género del Principado de Asturias (UTIGPA); Centro de Salud Mental I "La \\ Magdalena" (Área sanitaria III, Hospital Universitario San Agustín de Avilés. Asturias) y Facultad de psicología, \\ Universidad de Oviedo. \\ ***Centro de Salud Mental “La Ería” (Área sanitaria IV, Hospital Universitario Central de Asturias) y Universidad \\ Isabel I.
}

\section{Resumen}

Introducción: El acoso escolar es un fenómeno presente en todos los centros escolares y sus consecuencias sobre la salud mental de las víctimas pueden ser muy importantes. Las personas trans viven muchas veces con un estigma social que las hace más proclives a sufrir discriminación. El objetivo de este estudio es analizar las características del acoso escolar en personas trans y su relación con el autoconcepto, así como establecer una comparación con un grupo control cisgénero.

Método: La muestra estuvo compuesta por 68 participantes. El grupo experimental estuvo compuesto por 34 personas trans. El grupo control estuvo compuesto por 34 personas de características sociodemográficas similares.

Resultados: La prevalencia del acoso escolar es del 20,1\% en personas trans. No hay diferencias significativas en acoso escolar respecto al grupo control. Tampoco se encontraron diferencias respecto al autoconcepto ni otras variables sociodemográficas.

Correspondencia: Pablo Daniel Pérez Fernández, pablod.perezfdz@gmail.com, Facultad de psicología, Universidad de Oviedo. Plaza Feijoo, s/n, Oviedo. 
Conclusiones: Nuestros resultados no apoyan la idea, señalada por otros autores, de que las personas trans experimentan mayor acoso escolar ni un menor autoconcepto. Se ha hallado que reciben más agresiones basadas en objetos y maltrato físico que las personas cisgénero acosadas.

Palabras clave: acoso escolar; trans; autoconcepto; identidad de género, cisgénero.

\begin{abstract}
Introduction: Bullying is a phenomenon which is present in all schools and can have very important consequences on the victims' mental health. Trans people often live with a social stigma that makes them more prone to discrimination. The objective of this study is to analyze the characteristics of bullying in trans people and its relationship with self-concept, as well as to establish a comparison with a cisgender control group.

Method: The sample was made up of 68 participants. The experimental group was composed of 34 trans people. The control group comprised 34 people with similar sociodemographic characteristics.

Results: The prevalence of bullying is $20.1 \%$ in trans people. There are no significant differences in bullying when compared to the control group. There were also no differences regarding self-concept or other sociodemographic variables.

Conclusions: Our results do not support the claim made by other authors that trans people experience more bullying or less self-concept. It has been found that they receive more objectbased assaults and physical abuse than harassed cisgender people.

Keywords: bullying; trans; self-concept; gender identity; cisgender.
\end{abstract}

\title{
Introducción
}

El acoso escolar o bullying hace referencia a un tipo de violencia física o mental, o situación de maltrato prolongado y repetitivo por parte de un grupo o un sujeto que se dirige contra un individuo que no es capaz de defenderse y que tiene lugar en el contexto escolar (Cerezo, 2009; Díaz, 2015; Russell, Ryan, Toomey, Diaz \& Sánchez, 2011). La violencia escolar puede dirigirse hacia el profesorado, alumnado o propiedades y tiene lugar en las instalaciones del centro escolar, en los alrededores y en las actividades extraescolares (Serrano \& Iborra, 2005). La violencia se utiliza con la intención de causar dolor, humillación, vergüenza y abusos contra otra persona que está indefensa y que incluye, normalmente, un desequilibrio de poder, ya sea psicológico, físico, de estatus social o de otro rango (Collell \& Miquel, 2006). Para que este fenómeno se produzca, son necesarios dos sujetos bien diferenciados, la víctima y el agresor (Cerezo, 2009).

El aumento de la preocupación de la sociedad española por la violencia escolar llevó a la oficina del Defensor del Pueblo y al Comité Español de UNICEF a publicar en el año 2000 el primer informe sobre violencia escolar centrado en la incidencia del maltrato entre iguales a nivel estatal (Defensor del pueblo, 2000). Una réplica de este informe fue llevada a cabo en el 2007 por el mismo equipo de la Universidad Autónoma de Madrid (Defensor del Pueblo, 2007). Siguiendo a Garaigordobil y Oñederra (2010) la prevalencia y las características de una situación de acoso escolar no varían mucho 
de un país a otro, siendo, por tanto, un fenómeno generalizado en los países industrializados. Los estudios epidemiológicos muestran que la prevalencia del acoso escolar se encuentra entre el 20 y el $30 \%$.

En un estudio realizado en España se señala que uno de cada cinco alumnos se ha visto implicado en un proceso de acoso escolar y que es un fenómeno presente en cualquier clase social (Ayerbe et al., 2005). Otras investigaciones sitúan los índices de bullying en el 5,8\% en educación primaria y en el 3,8\% en secundaria (Garaigordobil \& Oñederra, 2010). En este mismo estudio también se señala que a medida que aumenta la edad disminuyen las conductas intimidatorias.

La investigación ha mostrado que las consecuencias de sufrir acoso durante la etapa escolar pueden ser importantes. Las víctimas de acoso escolar pueden sufrir síntomas depresivos, ansiedad y quejas psicosomáticas (Storch y Masia-Warner, 2004), altos niveles de estrés percibido (Estévez, Murgui, Musitu \& Moreno, 2008) y fracaso escolar (Ayerbe et al., 2005; Garaigordobil \& Oñederra, 2010).

Atendiendo a la violencia sufrida por la población trans, la transfobia, se refiere a las diferentes formas de violencia, odio, discriminación, rechazo, agresividad y actitudes negativas sobre las personas que transgreden o no encajan en las expectativas sociales de las normas de género (Platero, 2014). Este acoso y discriminación puede ser especialmente intenso en la adolescencia e inicios de la vida adulta.

La investigación que estudia la discriminación hacia las personas trans es bastante escasa (Hill \& Willoughby, 2005) así como la prevalencia y/o las características específicas del acoso escolar que sufren. Según la National Transgender Discrimination Survey Report (Grant et al., 2010), una encuesta acerca de la discriminación del colectivo trans en la que participaron un total de 6.456 personas trans y transgéneros con una edad de 18 a 89 años, de más de 50 estados, se señalan unas tasas de acoso escolar del 78\%. En estudios realizados en otros países, se señala que muchos jóvenes LGTB cambian sus planes de futuro respecto a la educación (Kosciw et al., 2014; Kosciw et al., 2016) y que quienes lo sufren obtienen pobres resultados académicos, aislamiento, menor motivación y son expulsados de la escuela (Formby, 2013; Jones \& Hillier, 2013; Kosciw et al., 2014; Kosciw et al., 2016). Las personas trans sufren mayores niveles de acoso que las personas cisgénero- aquellas cuya identificación se corresponde o concuerda con el género asignado al nacer (Factor \& Rothblum, 2007; Kosciw et al., 2014; Kosciw et al., 2016). Mientras unos estudios señalan que las Mujeres Trans (MT) suelen sufrir un mayor nivel de acoso que los Hombres Trans (HT) (Cano Oncala, 2004; Factor \& Rothblum, 2007), otros estudios han encontrado que los HT sufren más violencia en la escuela y otros contextos educativos que las MT (Grant et al., 2011). El acoso verbal es el tipo de acoso más frecuente en los ambientes educativo, laboral y familiar (Grant et al., 2011; Greytak, Kosciw, \& Diaz, 2009; Grossman \& D'Augelli, 2007). Además, la probabilidad de sufrir acoso disminuye cada año de retraso en hacer pública su identidad de género y las personas trans de mayor edad muestran menor nivel de acoso (Cano Oncala, 2004; Lombardi, Wilchins, Priesing, \& Malouf, 2001).

En el Eurobarómetro de 2019 elaborado por la Comisión Europea, un 58\% de la población española cree que la discriminación por motivos de identidad sexual se encuentra muy extendida en nuestra sociedad. Esta percepción, además, aumentó un 5\% respecto a la del Eurobarómetro del 2012, en el que un 53\% de la población espa- 
ñola creía que este tipo de discriminación se encontraba muy extendida. Esta idea es compartida por los adolescentes, que consideran que los trans son tratados de forma injusta por la sociedad y en los centros educativos (Colectivo de Lesbianas y Gays de la Safor, 2009).

En España, en el estudio realizado por Asenjo-Araque, Escolar-Gutiérrez, PascualSánchez, Ly-Pen y Becerra-Fernández (2017), se encontró que el 24,5\% de niños trans de la Unidad de Tratamiento de Identidad de Género de Madrid sufrió acoso escolar, de los que el 92,3\% señaló que se debía a la disforia de género, aunque no encontraron una relación significativa entre la disforia y el haber sufrido bullying. Asimismo, en la reciente investigación llevada a cabo por Devís-Devís et al. (2017), sobre discriminación a personas trans de edades comprendidas entre 10-62 años, se encontró que el contexto educativo es el segundo contexto en el que experimentan más acoso y que el tipo de maltrato que más sufren las personas trans es de tipo verbal.

El autoconcepto es entendido como el conjunto de percepciones que mantiene una persona sobre sí misma a partir de la valoración personal y de los otros significativos (Shavelson, Hubner, \& Stanton, 1976) así como el concepto que tiene de sí misma como un ser físico social y espiritual (García \& Musitu, 2014). Con respecto a su relación con el acoso escolar, se han hallado resultados contradictorios entre las diferentes dimensiones del autoconcepto para las personas agresoras, siendo las dimensiones académica y social las que han mostrado una relación más consistente con el fenómeno de la violencia escolar. Sin embargo, sí existe una relación clara entre el autoconcepto y el ser víctimas de acoso escolar, resultando en un autoconcepto pobre en todas las dimensiones (Boulton \& Smith, 1994; Rigby \& Slee, 1993).

\section{Método}

\section{Objetivos}

El objetivo del estudio es analizar las características y la prevalencia del acoso escolar en usuarios de la Unidad de Tratamiento de Identidad de Género del Principado de Asturias (UTIGPA) y establecer una comparación con un grupo control de personas cisgénero. Debido a las características de la muestra y de la población objeto de estudio y de forma similar a estudios previos (Devís-Devís et al.,2017), no se ha establecido un criterio de exclusión por edad.

\section{Población y Muestra}

El grupo experimental se compone de 34 usuarios trans que acuden a la UTIGPA. Sus edades se sitúan entre los 17-46 años. El 70,6\% son HT ( $n=24 ; M=24,58$ años; DT=8,480) y el 29,4\% son MT ( $n=10 ; M=23,40$ años; DT=5,441). Con respecto a la identidad de género para este grupo, era privada en el $79,4 \%(n=27)$ y para el $20,6 \%(n=7)$ pública. E1 5,9\% (n=2) tenían estudios primarios, el 38,3\% tenían estudios de ESO o FP1 ( $n=13)$, el $38,3 \%$ Bachiller o FP2 ( $n=13)$ y el 17,6\% $(n=6)$ estudios universitarios. 
El grupo control se compone de 34 personas con edades entre los 18-46 años, de las cuales el $29,4 \%$ son mujeres ( $n=10 ; M=23,20$ años; $D T=4,940)$ y el $70,6 \%$ restante son hombres ( $n=24 ; M=24,71$ años; DT=8,301). El 17,7\% tenían estudios de ESO o FP1 $(n=6)$, el 23,5\% Bachiller o FP2 ( $n=8)$ y el 58,8\% (n=20) estudios universitarios. No se encontraron diferencias significativas entre los grupos por edad ni por otras variables demográficas.

\section{Instrumento}

Además de la recogida de variables sociodemográficas, se administraron dos instrumentos: Cuestionario de Acoso entre iguales (CAI) y Autoconcepto Forma 5 (AF5).

El CAI de Magaz et al. (2016), uno de los pocos instrumentos desarrollados específicamente para evaluar la propia percepción de victimización de bullying. Está formado por las escalas: Conductas de Acoso (CAI-CA) de 39 ítems; Conductas Asociadas al Género (CAI-CAG) de 10 ítems; Escenarios (CAI-E) de 14 ítems; Personajes (CAI-P) de 9 ítems; Confidentes (CAI-C) de 6 ítems; Afrontamiento (CAI-A) de 9 ítems y; Estrés Postraumático (CAI-EP) de 11 ítems.

La escala de CAI-CA está compuesta por las subescalas de Maltrato verbal (11 ítems), Exclusión social directa (5 ítems), Amenazas (4 ítems), Ciberbullying (4 ítems), Exclusión social indirecta (4 ítems), Agresión basada en objetos (3 ítems) y Maltrato físico (8 ítems). La CAI-CAG está formada por 10 ítems, 5 para chicos y 5 para chicas. El contenido de los ítems específicos para chicos se refiere a ser objeto de maltrato verbal por atractivo físico o interesarse por cosas o actividades valoradas socialmente como femeninas mientras que, en el caso de las chicas, el contenido de los ítems se refiere a ser objeto de maltrato verbal por interesarse por actividades socialmente valoradas como masculinas, además de por la falta de atractivo físico. La CAI-CAG para chicas está compuesta por las subescalas Inconformismo de género y Atractivo físico. Tanto la escala de Conductas de Acoso (CAI-CA) como la escala de Conductas Asociadas al Género (CAI-CAG) poseen una escala de frecuencia de tres puntos que varía entre 1 («Nunca») y 3 («Muchas veces»). Respecto a la escala de Escenarios (CAI-E), está formada por las subescalas Escenarios situados fuera del recinto escolar (4 ítems), Escenarios no vigilados dentro del recinto escolar (4 ítems) y Escenarios vigilados dentro del recinto escolar (6 ítems). La escala de Personajes (CAI-P) se compone de las subescalas Defensores y observadores (3 ítems), Agresores de distintos cursos que la víctima (4 ítems) y Agresores del mismo curso que la víctima (2 ítems). En cuanto a la escala de Afrontamiento (CAI-A), ésta se compone de las subescalas Autofocalización negativa (4 ítems) y Focalización en el problema (3 ítems). Los datos sugieren que el CAI tiene unas propiedades psicométricas apropiadas y puede ser útil para la evaluación multidimensional de las conductas de acoso desde el punto de vista de la propia percepción de la victimización (Vera, Vélez y García, 2017). El cuestionario cuenta con una versión con ítems formulados en presente, que se utilizará con los usuarios menores de edad, mientras que para los usuarios mayores de edad se ha desarrollado una versión del mismo, con los ítems formulados en pasado.

El AF5 de García y Musitu (2014) está compuesto por 30 ítems con una escala de respuesta de 1 a 99. Este instrumento evalúa cinco dimensiones del autoconcepto: académica, social, emocional, familiar y física, con seis ítems cada una. Se trata de un 
instrumento de utilización clásica en España cuya estructura factorial ha sido confirmada por los autores (Bustos \& Galiana, 2015).

\section{Procedimiento de recogida y análisis de datos}

Tras obtener la autorización de la comisión de investigación del área sanitaria III del Hospital Universitario San Agustín de Avilés, se comenzó a recabar la colaboración voluntaria de los usuarios del grupo experimental, una vez obtenido el consentimiento informado. La selección de los participantes del grupo control se llevó a cabo mediante un muestreo de conveniencia, por el procedimiento de bola de nieve. Se creó una aplicación digital con los diferentes cuestionarios. Se aplicaron a diferentes entornos para acceder a una muestra de características similares a las del grupo experimental. La disforia de género se utilizó como criterio de exclusión.

El análisis de los datos fue realizado con el paquete estadístico IBM-SPSS (versión 24). Se realizó un análisis descriptivo para todas las variables recogidas. La normalidad de los datos se calculó a través de la prueba Kolmogorov-Smirnov. Se comprobó la homogeneidad de las varianzas mediante la prueba de Levene. Mediante pruebas de igualdad de medias $t$ de Student, se estudiaron las posibles diferencias existentes. En caso de significación estadística, el tamaño del efecto se comprobó mediante el coeficiente de determinación.

\section{Resultados}

Se presentan a continuación los análisis descriptivos de las distintas escalas del CAI Tablas $1,5,6,7$ y 8 ).

En la Tabla 1, se reflejan los resultados de la escala Personajes. Para el grupo trans, eran los chicos quienes más les agredían, tanto de su misma clase $(64,7 \%)$ como de cursos superiores al suyo (61,8\%). El 70,6\% contaba con un compañero que les defendiera y el $35,3 \%$ contaba con un adulto que les defendiera. El 76,5\% señala que había compañeros que se quedaban mirando sin hacer nada. El número de agresores en personas trans era de media 5,32 (DT=6,75) y el 70,6\% señalan que les acosaban con más compañeros. Para la mayor parte del grupo de cisgénero eran los chicos de su misma clase $(88,2 \%)$ quienes les agredían. El 50\% contaba con un compañero que les defendiera y el 32,4\% contaba con un adulto que les defendiera. El 91,2\% señala que había compañeros que se quedaban mirando sin hacer nada. El número de agresores en personas cisgénero era de media $3,88(\mathrm{DT}=5,575)$ y el $82,4 \%$ señalan que les acosaban con más compañeros.

$\mathrm{Si}$ atendemos de forma diferenciada al género, al grupo de HT les agredían tanto los chicos de su misma clase $(58,3 \%)$, de cursos superiores $(54,2 \%)$, como las chicas de su misma clase (50\%). El 70,8\% contaba con un compañero que les defendiera y el $25 \%$ contaba con un adulto que les defendiera. El 75\% señala que había compañeros que se quedaban mirando sin hacer nada. El número de agresores en los HT era de media 4,38 (DT=3,076) y el 70,8\% señalan que les acosaban con más compañeros. Para la mayor parte del grupo de hombres, eran los chicos de su misma clase (75\%) quienes les agredían. El 60,4\% contaba con un compañero que les defendiera y el $29,2 \%$ contaba con un adulto que les defendiera. El 85,4\% señala que había compañeros que 
se quedaban mirando sin hacer nada. El número de agresores en los hombres era de media 3,44 (DT=3,168) y el 75\% señalan que les acosaban con más compañeros.

Para el grupo MT eran los chicos quienes más les agredían, tanto de su misma clase (80\%) como de cursos superiores al suyo (80\%). El 70\% contaba con un compañero que les defendiera y el 60\% contaba con un adulto que les defendiera. El $80 \%$ señala que había compañeros que se quedaban mirando sin hacer nada. El número de agresores en las MT era de media 7,60 (DT=11,607) y el 70\% de las MT señalan que les acosaban con más compañeros. Para el grupo mujeres, eran los chicos $(80 \%)$ y las chicas $(75 \%)$ de su misma clase de quienes más les agredían. El 60\% contaba con un compañero que les defendiera y el $45 \%$ de mujeres contaba con un adulto que les defendiera. El $80 \%$ señala que había compañeros que se quedaban mirando sin hacer nada. El número de agresores en las mujeres era de media 7,40 (DT=9,923) y el $80 \%$ señalan que les acosaban con más compañeros.

Al grupo de hombres cis (HC) les agredían más los chicos de su misma clase $(91,7 \%)$. La mitad (50\%) contaba con un compañero que les defendiera y el 33,3\% contaba con un adulto que les defendiera. El 95,8\% señala que había compañeros que se quedaban mirando sin hacer nada y el 79,2\% señalan que les acosaban con más compañeros. El grupo mujeres cis (MC) era agredido mayoritariamente tanto por chicos $(80 \%)$ como por chicas $(100 \%)$ de su misma clase. La mitad (50\%) contaba con un compañero que les defendiera y solo el 30\% contaba con un adulto que les defendiera. El $80 \%$ señala que había compañeros que se quedaban mirando sin hacer nada y el $90 \%$ de las MC señalan que les acosaban con más compañeros.

Tabla 1

Datos descriptivos de la escala CAI-Personajes

\begin{tabular}{|c|c|c|c|c|c|c|c|c|}
\hline \multirow[b]{2}{*}{ CAI-P } & \multirow[b]{2}{*}{ Trans } & \multicolumn{5}{|c|}{ Muestra total } & \multirow[b]{2}{*}{$\mathrm{HC}$} & \multirow[b]{2}{*}{ MC } \\
\hline & & Cisgénero & Hombres & Mujeres & HT & MT & & \\
\hline & $\mathrm{n}(\%)$ & $\mathrm{n}(\%)$ & $\mathrm{n}(\%)$ & $\mathrm{n}(\%)$ & $\mathrm{n}(\%)$ & $\mathrm{n}(\%)$ & $\mathrm{n}(\%)$ & $\mathrm{n}(\%)$ \\
\hline $\begin{array}{l}\text { Chicos de mi misma } \\
\text { clase }\end{array}$ & $22(64,7)$ & $30(88,2)$ & $36(75,0)$ & $16(80)$ & $14(58,3)$ & $8(80)$ & $22(91,7)$ & $8(80)$ \\
\hline $\begin{array}{l}\text { Chicas de mi misma } \\
\text { clase }\end{array}$ & $17(50,0)$ & $18(52,9)$ & $20(41,7)$ & $15(75)$ & $12(50,0)$ & $5(50)$ & $8(33,3)$ & $10(100)$ \\
\hline $\begin{array}{l}\text { Chicos de cursos } \\
\text { superiores al mío }\end{array}$ & $21(61,8)$ & $18(52,9)$ & $26(54,2)$ & $13(65)$ & $13(54,2)$ & $8(80)$ & $13(54,2)$ & $5(50)$ \\
\hline $\begin{array}{l}\text { Chicas de cursos } \\
\text { superiores al mío }\end{array}$ & $10(29,4)$ & $9(26,5)$ & $14(29,2)$ & $5(25)$ & $8(33,3)$ & $2(20)$ & $6(25)$ & $3(30)$ \\
\hline $\begin{array}{l}\text { Chicos de cursos } \\
\text { inferiores al mío }\end{array}$ & $7(20,6)$ & $6(17,6)$ & $6(12,5)$ & $7(35)$ & $3(12,5)$ & $4(40)$ & $3(12,5)$ & $3(30)$ \\
\hline $\begin{array}{l}\text { Chicas de cursos } \\
\text { inferiores al mío }\end{array}$ & $7(20,6)$ & $6(17,6)$ & $6(12,5)$ & $7(35)$ & $3(12,5)$ & $4(40)$ & $3(12,5)$ & $3(30)$ \\
\hline $\begin{array}{l}\text { ¿Había algún } \\
\text { compañero que te } \\
\text { defendiera? }\end{array}$ & $24(70,6)$ & $17(50,0)$ & $29(60,4)$ & $12(60)$ & $17(70,8)$ & $7(70)$ & $12(50)$ & $5(50)$ \\
\hline $\begin{array}{l}\text { ¿Había algún adulto } \\
\text { que te defendiera? }\end{array}$ & $12(35,3)$ & $11(32,4)$ & $14(29,2)$ & $9(45)$ & $6(25,0)$ & $6(60)$ & $8(33,3)$ & $3(30)$ \\
\hline
\end{tabular}




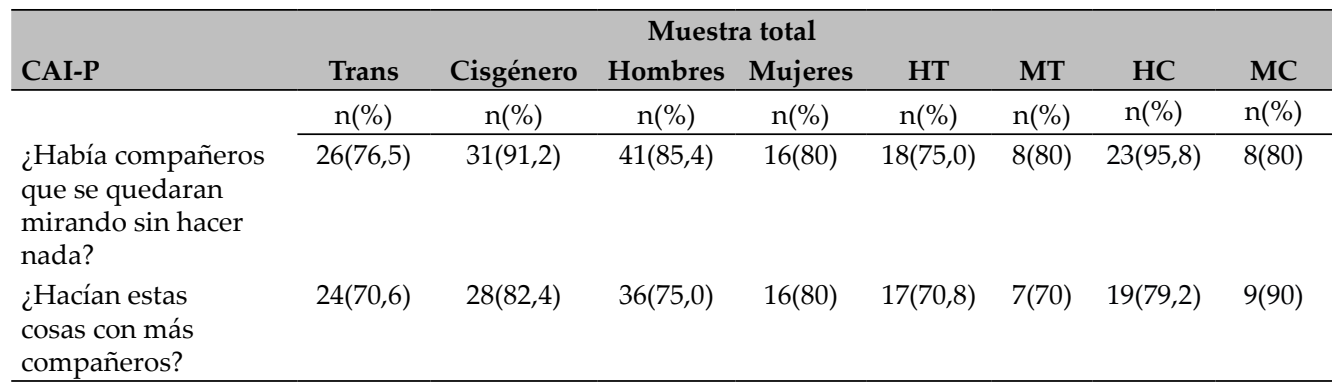

Nota: HT=Hombres Trans; $M T=$ Mujeres Trans; HC=Hombres Cis; $M C=$ Mujeres Cis.

En la Tabla 2 se reflejan los resultados de la escala Escenarios. Los HT sufrían el acoso en clase cuando no había profesorado en el $75 \%$, en la clase de gimnasia cuando no había profesorado en el 66,7\% y en los pasillos en un 66,7\%. Las MT sufrían el acoso en el patio cuando no vigilaba ningún profesor en el $90 \%$, en los pasillos en un $70 \%$, al salir de clase cerca del colegio en un $70 \%$, en la calle en un $70 \%$ y andando de camino a casa en un 70\%. El 82,4\% de las personas cisgénero sufrían el acoso en el patio cuando no vigilaba ningún profesor, el $76,5 \%$ en los pasillos y el $70,6 \%$ en clase cuando no había profesorado. El 70,6\% de personas trans sufrían el acoso en clase cuando no había profesorado, en los pasillos en el 67,6\% y el 67,6\% en el patio cuando no vigilaba ningún profesor.

Tabla2

Datos descriptivos de la escala CAI-Escenarios

\begin{tabular}{|c|c|c|c|c|c|c|c|c|}
\hline \multirow[b]{2}{*}{ CAI-E } & \multirow[b]{2}{*}{ Trans } & \multicolumn{6}{|c|}{ Muestra total } & \multirow[b]{2}{*}{ MC } \\
\hline & & Cisgénero & Hombres & Mujeres & HT & MT & $\mathrm{HC}$ & \\
\hline & $\mathrm{n}(\%)$ & $\mathrm{n}(\%)$ & $\mathrm{n}(\%)$ & $\mathrm{n}(\%)$ & $\mathrm{n}(\%)$ & $\mathrm{n}(\%)$ & $\mathrm{n}(\%)$ & $\mathrm{n}(\%)$ \\
\hline $\begin{array}{l}\text { En la clase de gim- } \\
\text { nasia cuando estaba } \\
\text { algún profesor/a }\end{array}$ & $13(38,2)$ & $13(38,2)$ & $16(33,3)$ & $10(50)$ & $8(33,3)$ & $5(50)$ & $8(33,3)$ & $5(50)$ \\
\hline $\begin{array}{l}\text { En las demás clases } \\
\text { cuando estaba algún } \\
\text { profesor/a }\end{array}$ & $14(41,2)$ & $16(47,1)$ & $20(41,7)$ & $10(50)$ & $9(37,5)$ & $5(50)$ & $11(45,8)$ & $5(50)$ \\
\hline $\begin{array}{l}\text { En la clase de gimna- } \\
\text { sia cuando no había } \\
\text { ningún profesor/a }\end{array}$ & $21(61,8)$ & $18(52,9)$ & $28(58,3)$ & $11(55)$ & $16(66,7)$ & $5(50)$ & $12(50)$ & $6(60)$ \\
\hline $\begin{array}{l}\text { En las demás clases } \\
\text { cuando no había } \\
\text { ningún profesor/a }\end{array}$ & $24(70,6)$ & $24(70,6)$ & $34(70,8)$ & $14(70)$ & $18(75,0)$ & $6(60)$ & $16(66,7)$ & $8(80)$ \\
\hline En los pasillos & $23(67,6)$ & $26(76,5)$ & $34(70,8)$ & $15(75)$ & $16(66,7)$ & $7(70)$ & $18(75)$ & $8(80)$ \\
\hline En el comedor & $7(20,6)$ & $9(26,5)$ & $11(22,9)$ & $5(25)$ & $6(25,0)$ & $1(10)$ & $5(20,8)$ & $4(40)$ \\
\hline En los aseos & $10(29,4)$ & $15(44,1)$ & $17(35,4)$ & $8(40)$ & $7(29,2)$ & $3(30)$ & $10(41,7)$ & $5(50)$ \\
\hline
\end{tabular}




\begin{tabular}{|c|c|c|c|c|c|c|c|c|}
\hline \multirow[b]{2}{*}{ CAI-E } & \multirow[b]{2}{*}{ Trans } & \multicolumn{6}{|c|}{ Muestra total } & \multirow[b]{2}{*}{ MC } \\
\hline & & Cisgénero & Hombres & Mujeres & HT & MT & HC & \\
\hline & $\mathrm{n}(\%)$ & $\mathrm{n}(\%)$ & $\mathrm{n}(\%)$ & $\mathrm{n}(\%)$ & $\mathrm{n}(\%)$ & $\mathrm{n}(\%)$ & $\mathrm{n}(\%)$ & $\mathrm{n}(\%)$ \\
\hline $\begin{array}{l}\text { En el patio cuando } \\
\text { vigilaba algún pro- } \\
\text { fesor }\end{array}$ & $14(41,2)$ & $20(58,8)$ & $20(41,7)$ & $14(70)$ & $8(33,3)$ & $6(60)$ & $12(50)$ & $8(80)$ \\
\hline $\begin{array}{l}\text { En el patio cuando } \\
\text { no vigilaba ningún } \\
\text { profesor }\end{array}$ & $23(67,6)$ & $28(82,4)$ & $32(66,7)$ & 19(95) & $14(58,3)$ & $9(90)$ & $18(75)$ & $10(100)$ \\
\hline $\begin{array}{l}\text { Al salir de clase, } \\
\text { cerca del colegio }\end{array}$ & $15(44,1)$ & $15(44,1)$ & $19(39,6)$ & $11(55)$ & $8(33,3)$ & $7(70)$ & $11(45,8)$ & $4(40)$ \\
\hline $\begin{array}{l}\text { En las actividades } \\
\text { extraescolares (na- } \\
\text { tación, fútbol, teatro, } \\
\text { etc.) }\end{array}$ & $11(32,4)$ & $12(35,3)$ & $14(29,2)$ & $9(45)$ & $6(25,0)$ & $5(50)$ & $8(33,3)$ & $4(40)$ \\
\hline En la calle & $15(44,1)$ & $22(64,7)$ & $24(50,0)$ & $13(65)$ & $8(33,3)$ & $7(70)$ & $16(66,7)$ & $6(60)$ \\
\hline En el autobús escolar & $7(20,6)$ & $8(23,5)$ & $8(16,7)$ & $7(35)$ & $3(12,5)$ & $4(40)$ & $5(20,8)$ & $3(30)$ \\
\hline $\begin{array}{l}\text { Andando de camino } \\
\text { a casa }\end{array}$ & $15(44,1)$ & $11(32,4)$ & $15(31,3)$ & $11(55)$ & $8(33,3)$ & $7(70)$ & $7(29,2)$ & $4(40)$ \\
\hline
\end{tabular}

Nota: HT=Hombres Trans; $M T=$ Mujeres Trans; HC=Hombres Cis; $M C=$ Mujeres Cis.

En la Tabla 3 se muestran los resultados de la escala Afrontamiento. El grupo de personas trans solía optar por no hacerles caso $(76,5 \%)$, evitar encontrarse a los compañeros que les acosaban $(61,8 \%)$ y pensar que ya se cansarían $(61,8 \%)$ y que ya dejarían de verles (61,8\%). El grupo de personas cisgénero solía optar por no hacerles caso $(79,4 \%)$, pensar que ya se cansarían $(73,5 \%)$ y que ya dejarían de verles $(70,6 \%)$ y evitar encontrarse a los compañeros que les acosaban $(67,6 \%)$.

Tabla 3

Datos descriptivos de la escala CAI- Afrontamiento.

\begin{tabular}{|c|c|c|c|c|c|c|c|c|}
\hline \multirow[b]{2}{*}{ CAI-A } & \multirow[b]{2}{*}{ Trans } & \multirow[b]{2}{*}{ Cisgénero } & \multicolumn{2}{|c|}{ Muestra total } & \multirow[b]{2}{*}{ HT } & \multirow[b]{2}{*}{ MT } & \multirow[b]{2}{*}{$\mathrm{HC}$} & \multirow[b]{2}{*}{ MC } \\
\hline & & & Hombres & Mujeres & & & & \\
\hline & $\mathrm{n}(\%)$ & $\mathrm{n}(\%)$ & $\mathrm{n}(\%)$ & $\mathrm{n}(\%)$ & $\mathrm{n}(\%)$ & $\mathrm{n}(\%)$ & $\mathrm{n}(\%)$ & $\mathrm{n}(\%)$ \\
\hline $\begin{array}{l}\text { Les respondían de } \\
\text { la misma forma que } \\
\text { ellos se metían con- } \\
\text { migo }\end{array}$ & $9(26,5)$ & $13(38,2)$ & $17(35,4)$ & $5(25)$ & $6(25,0)$ & $3(30)$ & $11(45,8)$ & $2(20)$ \\
\hline $\begin{array}{l}\text { Nada, porque creía } \\
\text { que esa situación } \\
\text { nunca se iba a solu- } \\
\text { cionar }\end{array}$ & $16(47,1)$ & $16(47,1)$ & $23(47,9)$ & $9(45)$ & $12(50,0)$ & $4(40)$ & $11(45,8)$ & $5(50)$ \\
\hline
\end{tabular}




\begin{tabular}{lcccccccc}
\hline \multicolumn{7}{c}{ CAI-A } & \multicolumn{7}{c}{ Muestra total } & & & & \\
& Trans & Cisgénero & Hombres & Mujeres & HT & MT & HC & MC \\
\cline { 2 - 8 } & $\mathrm{n}(\%)$ & $\mathrm{n}(\%)$ & $\mathrm{n}(\%)$ & $\mathrm{n}(\%)$ & $\mathrm{n}(\%)$ & $\mathrm{n}(\%)$ & $\mathrm{n}(\%)$ & $\mathrm{n}(\%)$ \\
\hline $\begin{array}{l}\text { Evitaba encontrarme } \\
\text { a los compañeros }\end{array}$ & $21(61,89$ & $23(67,6)$ & $29(60,4)$ & $15(75)$ & $13(54,2)$ & $8(80)$ & $16(66,7)$ & $7(70)$ \\
$\begin{array}{l}\text { que me pegaban, se } \\
\text { burlaban o se metían } \\
\text { conmigo }\end{array}$ & & & & & & & & \\
$\begin{array}{l}\text { Pasaba de ellos, no } \\
\text { les hacía caso }\end{array}$ & $26(76,5)$ & $27(79,4)$ & $35(72,9)$ & $18(90)$ & $16(66,7)$ & $10(100)$ & $19(79,2)$ & $8(80)$ \\
$\begin{array}{l}\text { Pensaba que ya se } \\
\text { cansarían }\end{array}$ & $21(61,8)$ & $25(73,5)$ & $32(66,7)$ & $14(70)$ & $14(58,3)$ & $7(70)$ & $18(75)$ & $7(70)$ \\
$\begin{array}{l}\text { Pensaba que ya } \\
\text { dejaría de verles }\end{array}$ & $21(61,8)$ & $24(70,6)$ & $31(64,6)$ & $14(70)$ & $13(54,2)$ & $8(80)$ & $18(75)$ & $6(60)$ \\
$\begin{array}{l}\text { Pensaba o le pedí a } \\
\text { mis padres que me } \\
\text { cambiaran de colegio }\end{array}$ & $8(23,5)$ & $7(20,6)$ & $9(18,8)$ & $6(30)$ & $5(20,8)$ & $3(30)$ & $4(16,7)$ & $3(30)$ \\
$\begin{array}{l}\text { Me apunté a clases } \\
\text { para aprender a } \\
\text { defenderme (karate, }\end{array}$ & $6(17,6)$ & $5(14,7)$ & $9(18,8)$ & $2(10)$ & $4(16,7)$ & $2(20)$ & $5(20,8)$ & $0(0)$ \\
judo, etc.)
\end{tabular}

Nota: HT=Hombres Trans; MT= Mujeres Trans; HC=Hombres Cis; MC= Mujeres Cis.

En la Tabla 4 se presentan los resultados para la escala Confidentes. El 44,1\% de las personas trans solía hablar del acoso con sus compañeros o amigos de clase, el 35,3\% con su madre y un 35,3\% no hablaba con nadie. El 55,9\% de las personas cisgénero hablaba del acoso con sus compañeros o amigos de clase, el 32,4\% con su madre y un $41,2 \%$ no hablaba con nadie.

Tabla 4

Datos descriptivos de la escala CAI- Confidentes

\begin{tabular}{lcccccccc}
\hline \multirow{2}{*}{ CAI-C } & Trans & Cisgénero & \multicolumn{2}{c}{$\begin{array}{c}\text { Muestra total } \\
\text { Hombres Mujeres }\end{array}$} & HT & MT & HC & MC \\
\hline & $\mathrm{n}(\%)$ & $\mathrm{n}(\%)$ & $\mathrm{n}(\%)$ & $\mathrm{n}(\%)$ & $\mathrm{n}(\%)$ & $\mathrm{n}(\%)$ & $\mathrm{n}(\%)$ & $\mathrm{n}(\%)$ \\
\cline { 2 - 8 } Con tu padre & $4(11,8)$ & $7(20,6)$ & $7(14,6)$ & $4(20)$ & $2(8,3)$ & $2(20)$ & $5(20,8)$ & $2(20)$ \\
Con tu madre & $12(35,3)$ & $11(32,4)$ & $15(31,3)$ & $8(40)$ & $6(25,0)$ & $6(60)$ & $9(37,5)$ & $2(20)$ \\
Con tus hermanos & $5(14,7)$ & $5(14,7)$ & $7(14,6)$ & $3(15)$ & $2(8,3)$ & $3(30)$ & $5(20,8)$ & $0(0)$ \\
$\begin{array}{l}\text { Con tus compañeros } \\
\text { o amigos de clase }\end{array}$ & $15(44,1)$ & $19(55,9)$ & $23(47,9)$ & $11(55)$ & $10(41,7)$ & $5(50)$ & $13(54,2)$ & $6(60)$ \\
$\begin{array}{l}\text { Con otros amigos de } \\
\text { la calle o barrio }\end{array}$ & $8(23,5)$ & $8(23,5)$ & $13(27,1)$ & $3(15)$ & $6(25,0)$ & $2(20)$ & $7(29,2)$ & $1(10)$ \\
$\begin{array}{l}\text { Con nadie, me lo } \\
\text { callaba }\end{array}$ & $12(35,3)$ & $14(41,2)$ & $21(43,8)$ & $5(25)$ & $10(41,7)$ & $2(20)$ & $11(45,8)$ & $3(30)$ \\
\hline
\end{tabular}


En la Tabla 5 se reflejan los resultados de la escala Estrés Postraumático. Las personas trans señalan como síntomas más frecuentes: tensión $(64,7 \%)$, pensamientos intrusivos $(44,1 \%)$, malestar $(44,1 \%)$ y apatía $(41,2 \%)$. Dentro del grupo control, los síntomas más frecuentes que señalan son irritabilidad (52,9\%), menos ganas de ver a sus amigos (52,9\%), tensión (50\%) y malestar (47,1\%). En los HT, los síntomas más frecuentes que señalan son tensión (66,7\%), malestar (45,8\%) e irritabilidad (41,7\%). En las MT, los síntomas más frecuentes que señalan son tensión $(60 \%)$, pensamientos intrusivos $(60 \%)$ y apatía $(50 \%)$.

Tabla 5

Datos descriptivos de la escala Estrés Postraumático (CAI-EP)

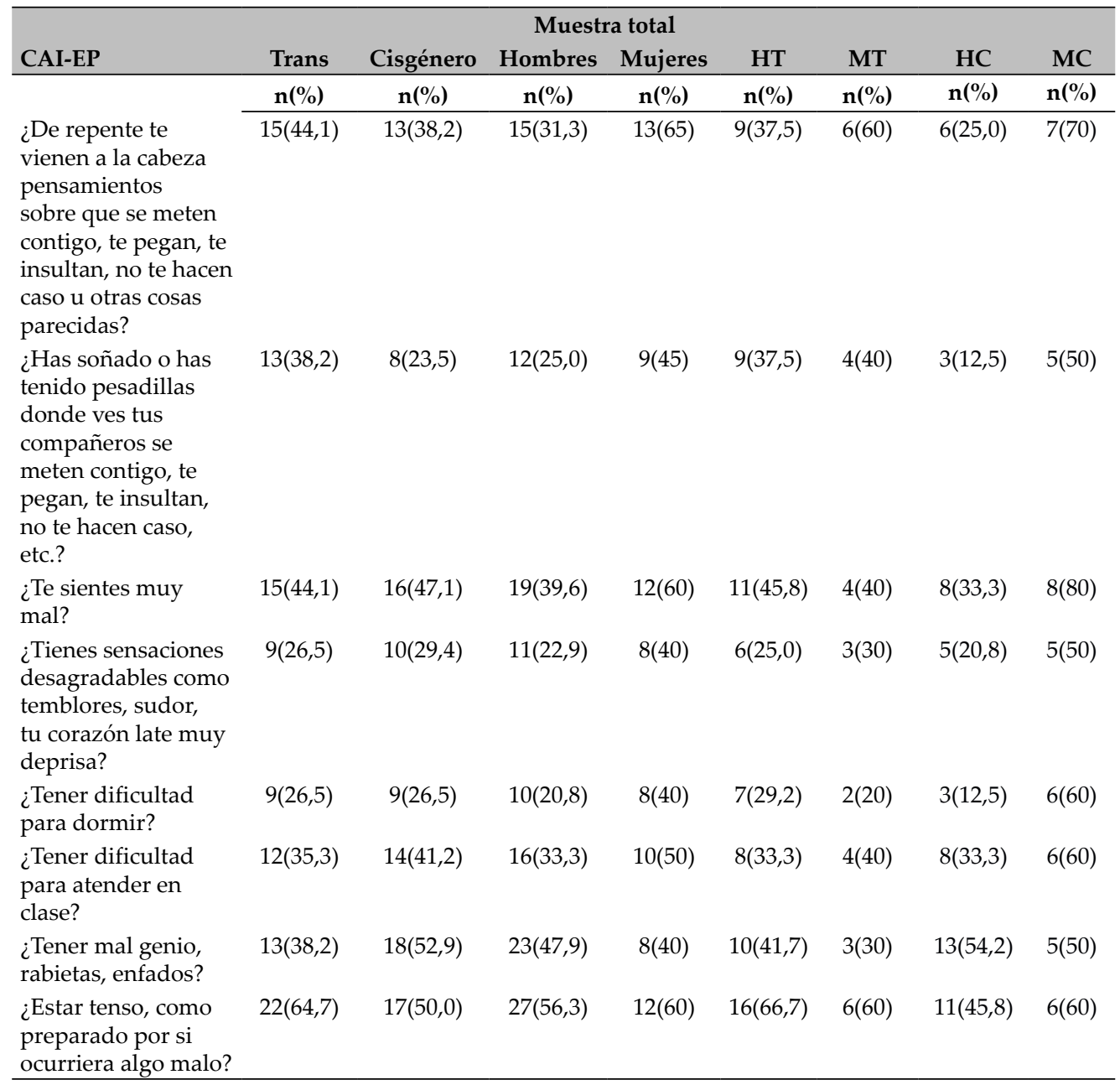




\begin{tabular}{|c|c|c|c|c|c|c|c|c|}
\hline \multirow[b]{2}{*}{ CAI-EP } & \multirow[b]{2}{*}{ Trans } & \multicolumn{5}{|c|}{ Muestra total } & \multirow[b]{2}{*}{ HC } & \multirow[b]{2}{*}{ MC } \\
\hline & & Cisgénero & Hombres & Mujeres & HT & MT & & \\
\hline & $n(\%)$ & $n(\%)$ & $n(\%)$ & $n(\%)$ & $n(\%)$ & $n(\%)$ & $\mathrm{n}(\%)$ & $\mathrm{n}(\%)$ \\
\hline $\begin{array}{l}\text { ¿Tener menos ganas } \\
\text { de ver a tus amigos? }\end{array}$ & $9(26,5)$ & $18(52,9)$ & $18(37,5)$ & $9(45)$ & $7(29,2)$ & $2(20)$ & $11(45,8)$ & $7(70)$ \\
\hline $\begin{array}{l}\text { ¿Tener menos ganas } \\
\text { de jugar o hacer } \\
\text { cosas que antes te } \\
\text { gustaba hacer? }\end{array}$ & $14(41,2)$ & $15(44,1)$ & $19(39,6)$ & $10(50)$ & $9(37,5)$ & $5(50)$ & $10(41,7)$ & $5(50)$ \\
\hline $\begin{array}{l}\text { ¿Intentar no pensar } \\
\text { en los sitios donde } \\
\text { te ocurren estas } \\
\text { cosas? }\end{array}$ & $13(38,2)$ & $15(44,1)$ & $17(35,4)$ & $11(55)$ & $9(37,5)$ & $4(40)$ & $8(33,3)$ & $7(70)$ \\
\hline
\end{tabular}

Nota: HT=Hombres Trans; $M T=$ Mujeres Trans; HC=Hombres Cis; $M C=$ Mujeres Cis.

A continuación, se muestran los descriptivos para las distintas subescalas de la escala Conductas de Acoso. No existen diferencias significativas entre grupo experimental y control. (Tabla 6).

Tabla 6

Medias de las puntuaciones en subescalas de la escala CAI-CA y prueba t de Student

\begin{tabular}{lccccccc}
\hline \multicolumn{7}{c}{ Trans $\mathbf{( N = 3 4 )}$} & \multicolumn{2}{c}{ Cisgénero $\mathbf{( N = 3 4 )}$} & & & \\
\hline & $\mathrm{M}$ & $\mathrm{DT}$ & $\mathrm{M}$ & $\mathrm{DT}$ & $\mathrm{t}$ & $\mathrm{gl}$ & $\mathrm{p}$ \\
\hline Maltrato verbal & 19,29 & 5,64 & 18,82 & 6,23 & 0,32 & 66 & 0,51 \\
$\begin{array}{l}\text { Exclusión social } \\
\text { directa }\end{array}$ & 8,94 & 3,44 & 7,97 & 3,21 & 1,20 & 66 & 0,31 \\
Amenazas & 5,76 & 1,84 & 5,35 & 1,95 & 0,89 & 66 & 0,85 \\
$\begin{array}{l}\text { Ciberbullying } \\
\text { Exclusión social }\end{array}$ & 4,79 & 1,12 & 4,59 & 1,01 & 0,79 & 66 & 0,33 \\
$\begin{array}{l}\text { indirecta } \\
\text { Agresión basada } \\
\text { en objetos }\end{array}$ & 6,58 & 2,45 & 6,17 & 2,50 & 0,68 & 66 & 0,92 \\
$\begin{array}{l}\text { Maltrato físico } \\
\text { CAI-C }\end{array}$ & 11,61 & 4,57 & 10,64 & 2,38 & 1,09 & 49,72 & 0,27 \\
\hline
\end{tabular}

Nota: $M=$ media; DT=desviación típica; $t$ : valor de la $t$ de Student; gl: grados de libertad.

Se realizó un análisis de frecuencias para analizar la prevalencia de acoso escolar y de los diferentes tipos de acoso. Se seleccionó únicamente a las personas que habían sufrido acoso escolar, tomando como punto de corte la puntuación media de la escala CAI-CA. La prevalencia de acoso escolar en personas trans fue del $20,1 \%$ y en cisgénero del 17,6\% (Tabla 7). 
Tabla 7

Análisis de frecuencias de la escala Conductas de Acoso (CAI-CA)

\begin{tabular}{lccc}
\hline & Trans (N=7) & Cisgénero (N=6) & Total \\
\hline & $\mathrm{n}(\%)$ & $\mathrm{n}(\%)$ & $\mathrm{n}(\%)$ \\
Maltrato verbal & $7(100)$ & $6(100)$ & $13(100)$ \\
Exclusión social directa & $5(71,4)$ & $6(100)$ & $11(84,6)$ \\
Amenazas & $3(42,9)$ & $3(50)$ & $6(46,2)$ \\
Ciberbullying & $0(0)$ & $0(0)$ & $0(0)$ \\
Exclusión social indirecta & $3(42,9)$ & $4(66,7)$ & $7(53,8)$ \\
Agresión basada en objetos & $7(100)$ & $1(16,7)$ & $8(61,5)$ \\
Maltrato físico & $5(71,4)$ & $0(0)$ & $5(38,5)$ \\
Acoso Escolar & $7(20,1)$ & $6(17,6)$ & $13(19,1)$ \\
\hline
\end{tabular}

Se llevó a cabo una comparación de medias entre las subescalas del CAI-CA, seleccionando únicamente a las personas que fueron víctimas de acoso escolar, encontrando diferencias significativas en las subescalas de agresión basada en objetos y maltrato físico (Tabla 8).

Tabla 8

Medias de las puntuaciones en subescalas de la escala CAI-C y prueba $T$

\begin{tabular}{lccccccccccc}
\hline & \multicolumn{1}{c}{ Trans $\mathbf{( N = 7 )}$} & \multicolumn{2}{c}{$\begin{array}{c}\text { Cisgénero } \\
(\mathbf{N = 6})\end{array}$} & \multicolumn{2}{c}{$\begin{array}{c}\text { Prueba de } \\
\text { Levene }\end{array}$} & \multicolumn{2}{c}{ Prueba t } & & \\
\hline & $\mathrm{M}$ & $\mathrm{DT}$ & $\mathrm{M}$ & $\mathrm{DT}$ & $\mathrm{F}$ & $\mathrm{Sig}$. & $\mathrm{t}$ & $\mathrm{gl}$ & $\mathrm{p}$ & $\mathrm{r}$ \\
$\begin{array}{l}\text { Agresión } \\
\text { basada en } \\
\text { objetos }\end{array}$ & 8,42 & 0,78 & 5,00 & 1,67 & 7,40 & 0,02 & 5,40 & 5 & 0,02 & 0.62 \\
$\begin{array}{l}\text { Maltrato } \\
\text { físico }\end{array}$ & 19,14 & 4,48 & 13,16 & 2,38 & 22,56 & 0,00 & 3,87 & 6 & 0,01 & 0.40 \\
\hline
\end{tabular}

Nota: $M=$ media; $D T=$ desviación típica; $t=$ valor de la $t$ de Student; $g l=$ grados de libertad; $r=$ tamaño del efecto.

Seleccionando a las personas que sufrieron acoso escolar, se exploró la posible influencia de las variables sociodemográficas en las escalas de ambos cuestionarios, pero no se encontraron diferencias significativas en ninguna de ellas $(p>0,05)$.

Analizando los ítems de este cuestionario de forma independiente, no se encontraron diferencias entre el grupo control y el grupo experimental. Sin embargo, sí se encontraron diferencias significativas al comparar las puntuaciones entre mujeres y hombres y entre HT y MT en algunos ítems. (Tabla 9) 
Tabla 9

Medias de las puntuaciones en ítems de la escala CAI-CA y prueba $T$

\begin{tabular}{|c|c|c|c|c|c|c|c|c|c|c|}
\hline & \multicolumn{2}{|c|}{ Hombres $(n=48)$} & \multicolumn{2}{|c|}{$\begin{array}{l}\text { Mujeres } \\
(\mathrm{n}=20)\end{array}$} & \multicolumn{2}{|c|}{$\begin{array}{l}\text { Prueba de } \\
\text { Levene }\end{array}$} & \multicolumn{4}{|c|}{ Prueba $t$} \\
\hline & M & DT & M & DT & $\mathrm{F}$ & Sig & $\mathrm{t}$ & gl & $\mathrm{p}$ & $\mathrm{r}$ \\
\hline $\begin{array}{l}\text { Me rompían } \\
\text { cosas }\end{array}$ & 1,21 & 0,50 & 1,70 & 0,80 & 13,59 & 0,00 & $-2,54$ & 25,48 & ,01 & 0.11 \\
\hline \multirow[t]{3}{*}{$\begin{array}{l}\text { Me ponían la } \\
\text { zancadilla }\end{array}$} & 1,25 & 0,48 & 1,70 & 0,73 & 9,09 & 0,00 & $-2,52$ & 26,18 & ,01 & 0.11 \\
\hline & \multicolumn{2}{|l|}{ HT (N=24) } & \multicolumn{2}{|c|}{ MT $(\mathrm{N}=10)$} & \multicolumn{2}{|c|}{$\begin{array}{l}\text { Prueba de } \\
\text { Levene }\end{array}$} & \multicolumn{4}{|c|}{ Prueba $t$} \\
\hline & M & DT & M & DT & $\mathrm{F}$ & Sig & $\mathrm{t}$ & $\mathrm{gl}$ & $\mathrm{p}$ & $\mathrm{r}$ \\
\hline $\begin{array}{l}\text { Me pegaban } \\
\text { patadas }\end{array}$ & 1,13 & 0,448 & 1,80 & 0,78 & 7,83 & 0,00 & $-2,54$ & 11,50 & ,02 & 0.21 \\
\hline
\end{tabular}

Nota: $M=$ media; $D T=$ desviación típica; $t=$ valor de la $t$ de Student; $g l=$ grados de libertad; $r=$ tamaño del efecto.

No existen diferencias estadísticamente significativas entre los grupos control y experimental en la escala CAI-CAG, encontrándose diferencias significativas en la CAI-CAG de chicos y en la CAI-CAG de chicas (Tabla 10).

Tabla 10

Comparación de Medias de las puntuaciones en el cuestionario CAI-CAG

\begin{tabular}{|c|c|c|c|c|c|c|c|c|c|c|c|}
\hline \multicolumn{2}{|c|}{ CAI-CAG } & \multicolumn{2}{|c|}{ HT (N=24) } & \multirow{2}{*}{$\frac{\mathbf{M T}(\mathbf{N}=\mathbf{1 0})}{\mathrm{DT}}$} & \multicolumn{3}{|c|}{$\begin{array}{c}\text { Prueba de } \\
\text { Levene }\end{array}$} & \multicolumn{4}{|c|}{ Prueba $t$} \\
\hline & $\mathrm{M}$ & DT & M & & F & Sig. & $\mathrm{t}$ & gl & & $\mathrm{p}$ & r \\
\hline Chico & 6,54 & 1,88 & 11,2 & 3,25 & 4,87 & 0,03 & 4,23 & 11,60 & 0,03 & 0.50 & \\
\hline Chica & 9,66 & 3,34 & 5,9 & 1,52 & 8,78 & 0,00 & 4,50 & 31,58 & 0,00 & 0.33 & \\
\hline
\end{tabular}

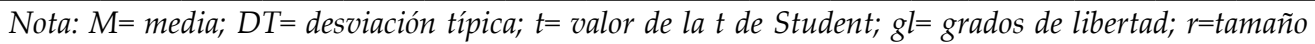
del efecto.

No se hallaron diferencias estadísticamente significativas entre los grupos control y experimental en la escala AF5. En la Tabla 11 se exponen los resultados. Analizando los ítems del cuestionario por separado tampoco se encontraron diferencias significativas.

Se exploró la influencia que pudieran tener las variables sociodemográficas (edad, nivel de estudios, razón sexo-género, rol de género que desempeñaba durante su etapa escolar) en las escalas de ambos cuestionarios, pero no se encontraron diferencias significativas en ninguna de ellas $(p>0,05)$. 
Tabla 11

Medias de las puntuaciones en el cuestionario AF5 y prueba T

\begin{tabular}{|c|c|c|c|c|c|c|c|c|c|}
\hline \multirow[t]{2}{*}{ AF5 } & \multicolumn{2}{|c|}{$\begin{array}{l}\text { Trans } \\
(\mathrm{N}=34)\end{array}$} & \multicolumn{2}{|c|}{$\begin{array}{l}\text { Cisgénero } \\
(\mathrm{N}=34)\end{array}$} & $\begin{array}{l}\text { Prueba de } \\
\text { Levene }\end{array}$ & \multicolumn{2}{|c|}{ Prueba $t$} & \multirow[b]{2}{*}{ gl } & \multirow[b]{2}{*}{$\mathrm{p}$} \\
\hline & M & DT & M & DT & $\mathrm{F}$ & Sig & $t$ & & \\
\hline $\begin{array}{l}\text { Autoconcepto } \\
\text { Social }\end{array}$ & 5,82 & 2,1 & 5,92 & 3,08 & 4,04 & 0,05 & $-0,15$ & 59,52 & 0,87 \\
\hline $\begin{array}{l}\text { Autoconcepto } \\
\text { Académico/ } \\
\text { Laboral }\end{array}$ & 6,67 & 2,20 & 7,21 & 1,70 & 0,69 & 0,40 & $-1,14$ & 66 & 0,40 \\
\hline $\begin{array}{l}\text { Autoconcepto } \\
\text { Emocional }\end{array}$ & 4,96 & 2,41 & 5,71 & 2,34 & 0,18 & 0,67 & $-1,29$ & 66 & 0,67 \\
\hline $\begin{array}{l}\text { Autoconcepto } \\
\text { Familiar }\end{array}$ & 7,45 & 2,28 & 6,96 & 2,45 & 0,00 & 0,97 & 0,85 & 66 & 0,97 \\
\hline $\begin{array}{l}\text { Autoconcepto } \\
\text { Físico }\end{array}$ & 4,60 & 2,24 & 4,78 & 2,30 & 0,00 & 0,93 & $-0,34$ & 66 & 0,93 \\
\hline
\end{tabular}

Nota: $M=$ media; $D T=$ desviación típica; $t=$ valor de la $t$ de Student; $g l=$ grados de libertad.

\section{Discusión y conclusiones}

El objetivo principal de este estudio era analizar la prevalencia y las características del acoso escolar entre los usuarios de la UTIGPA y establecer una comparación con un grupo control de personas cisgénero de características sociodemográficas similares. Los resultados obtenidos en este estudio contrastan con las tasas de mayor discriminación hacia las personas trans encontradas en la literatura (Factor \& Rothblum, 2007; Formby, 2013; Jones \& Hillier, 2013; Kosciw et al., 2014; Kosciw et al., 2016). En nuestra muestra no se hallaron diferencias significativas respecto al grupo control. Este resultado también se halló en el estudio sobre acoso escolar realizado en la UTIG de Madrid (Asenjo-Araque et al., 2017). La ausencia de diferencias en los niveles de acoso podría deberse a discrepancias culturales en la aceptación de la diversidad de género, así como al hecho de que la muestra fue extraída de una unidad en la que las personas trans cuentan con el apoyo psicológico de dos profesionales de la salud mental, al tamaño de la muestra y a que la mayor parte de personas no habían llegado a expresar de manera pública su identidad de género.

Las prevalencias halladas, tanto para personas trans como para el total de la muestra, coinciden con las encontradas en la literatura en la población general (Garaigordobil \& Oñederra, 2010).

Nuestros resultados sugieren, con respecto a los personajes que intervienen en el acoso escolar, que son los chicos los que más agreden tanto a las personas cisgénero como a las trans de ambos géneros. Con la particularidad de que a las personas trans 
les acosaban los chicos de cursos superiores al suyo además de los de su misma clase, especialmente a las MT. Esto apoya los resultados de otros estudios que señalan que los agresores suelen ser varones y de igual o mayor edad que las personas a las que agreden (Ayerbe et al., 2005; Cerezo, 2006, 2009; Defensor del pueblo, 2000; Defensor del Pueblo, 2007; Garaigordobil \& Oñederra, 2010).

En términos generales, la mayoría de las personas de nuestra muestra contaba con algún compañero que les defendiera, pero pocas veces recibían el apoyo de un adulto. La gran mayoría de los compañeros no intervinieron cuando presenciaban una situación de acoso y los agresores solían actuar en grupo, siendo mayor el número de agresores en las personas trans, especialmente en las MT.

En cuanto al escenario en el que se produce el acoso, se han obtenido datos variados. Por una parte, en la mayoría de personas cisgénero y de MT, el acoso se producía en el patio del colegio cuando no vigilaba el profesorado, en concordancia con los resultados hallados por otros estudios en España con población general (Garaigordobil y Oñederra, 2010). El escenario de acoso más frecuente para personas trans y HT era el aula cuando no había profesorado, como se encontró en los estudios a nivel estatal (Defensor del pueblo, 2000; Defensor del Pueblo, 2007).

En lo referente a la forma de afrontar el problema, la gran mayoría de personas opta por estrategias de autofocalización negativa, como no hacerles caso, pensar que ya se cansarían y que dejarían de verles o evitar encontrarse a los compañeros que les acosaban, como apuntan los resultados hallados en los perfiles de las víctimas de acoso escolar (Cerezo, 2006, 2009).

Nuestros resultados sobre los confidentes no coinciden con los encontrados en la literatura, que señalan que la mayor parte de las personas que sufren acoso escolar no hablan con nadie (Defensor del pueblo, 2000; Defensor del Pueblo, 2007), optando la mayoría de las personas de la muestra por hablar con compañeros de clase. Esto es así en toda la muestra excepto en las MT, quienes se lo cuentan a su madre además de a sus compañeros. Esta diferencia quizás explique el gran número de personas de la muestra que cuenta con un compañero que les defendiera.

Respecto a los síntomas referidos a raíz del acoso escolar vemos que, dentro del grupo experimental, los síntomas más frecuentes que señalan son tensión, pensamientos intrusivos, malestar y apatía, presentando los HT en mayor proporción irritabilidad. Todos ellos coinciden con los que se encuentran en la literatura española e internacional (Estévez et al., 2008; Formby, 2013; Jones \& Hillier, 2013; Kosciw et al., 2014; Kosciw et al., 2016; Storch \& Masia-Warner, 2004).

Los dos tipos de acoso más frecuentes encontrados en el total de muestra son el maltrato verbal y la exclusión social, coincidiendo plenamente con los resultados encontrados en estudios realizados en España y otros países (Defensor del pueblo, 2000; Defensor del Pueblo, 2007; Devís-Devís et al., 2017; Garaigordobil \& Oñederra, 2010; Grant et al., 2011; Greytak et al., 2009; Grossman \& D'Augelli, 2007). Respecto a las personas trans acosadas, además del maltrato verbal y la exclusión social, encontramos diferencias significativas respecto a los cisgénero en la agresión basada en objetos y el maltrato físico.

Se encontraron diferencias estadísticamente significativas respecto a las conductas de acoso asociadas al género. Los HT sufren conductas de acoso asociadas al género 
femenino y las MT conductas de acoso asociadas al género masculino. Es decir, las personas trans son agredidas en cuanto a su rol de género. En nuestro estudio se halló que las MT sufrían más agresiones físicas en forma de patadas, rotura de objetos personas y zancadillas que los HT, datos que coinciden con los resultados de unos estudios (Cano Oncala, 2004; Factor \& Rothblum, 2007), en contradicción con otras investigaciones que señalan que los HT reciben más acoso (Grant et al., 2010).

En nuestro estudio no se encontraron diferencias significativas en los diferentes instrumentos entre las muestras, en ninguna de las variables sociodemográficas evaluadas, contradiciendo esto lo encontrado en algunos estudios (Cano Oncala, 2004; Factor \& Rothblum, 2007; Grant et al., 2011; Lombardi et al., 2001). Esto datos pueden deberse a la no diferencia entre los niveles de acoso entre personas trans y cisgénero, puesto que, si no se han encontrado diferencias significativas en esta variable, es esperable que no haya diferencias en el autoconcepto derivadas del acoso. Sin embargo, nuestros datos están limitados por el reducido tamaño de muestra y no podrían generalizarse a toda la población trans. Si bien nuestros resultados no parecen corroborar un mayor acoso escolar en las personas trans de nuestro estudio, creemos necesario continuar investigando la relación que pueda existir entre el rol de género que tienen las personas trans y su identidad de género, ya que en nuestra muestra la mayor parte de personas no habían llegado a expresar de manera pública su identidad de género. Los nada desdeñables porcentajes de personas trans que refieren síntomas de ansiedad y depresión ponen de manifiesto las graves consecuencias que pueden tener las experiencias de acoso escolar sobre la vida de las personas trans y, por ende, la importancia de que los profesionales de la salud mental y de la educación estén preparados para poner en marcha estrategias terapéuticas específicas para este fenómeno.

\section{Referencias}

Asenjo-Araque, N., Escolar-Gutiérrez, M. C., Pascual-Sánchez, A., Ly-Pen, D., \& BecerraFernández, A. (2017). Bullying victimization in variant gender children: Experience in Madrid's gender identity unit. Sexual Health Issues, 1(1), 1-3. doi:10.15761/ SHI.1000103

Ayerbe, E., Aramendi, J., Balsells, B., Miñambres, A., Arco, I., \& Armengol, C. (2005). Estudio comparativo entre el País Vasco y Cataluña sobre la indisciplina y la violencia escolar. Universidad Del País Vasco, Universidad de Lleida: UAB.

Boulton, M. J., \& Smith, P. K. (1994). Bully/victim problems in middle-school children: Stability, self-perceived competence, peer perceptions and peer acceptance. British Journal of Developmental Psychology, 12(3), 315-329. doi:10.1111/j.2044-835X.1994. tb00637.x

Bustos, V., Oliver, A., \& Galiana, L. (2015). Validación del Autoconcepto Forma 5 en Universitarios Peruanos: Una Herramienta para la Psicología Positiva. Psicologia: Reflexão E Crítica, 28(4), 690-697. https://doi.org/10.1590/1678-7153.201528406

Cano Oncala, G. (2004). La construcción de la identidad de género en pacientes trans. Revista de La Asociación Española de Neuropsiquiatría, 21, 21-30. Retrieved from http:// scielo.isciii.es/scielo.php?pid=S0211-57352004000100003\&script=sci_arttext 
Cerezo, F. (2006). Análisis comparativo de variables socio-afectivas diferenciales entre los implicados en bullying. Estudio de un caso de víctima-provocador. Anuario de Psicología Clínica Y de La Salud,2, 27-34.

Cerezo, F. (2009). Bullying: análisis de la situación en las aulas españolas. International Journal of Psychology and Psychological Therapy, 9(3), 367-378.

Colectivo de Lesbianas y Gays de la Safor-CLGS (2009). Homofobia y transfobia en el ámbito educativo. Gandía: CLGS.

Collell, J., \& Miquel, C. E. (2006). El acoso escolar: un enfoque psicopatológico. Anuario de Psicología Clínica Y de La Salud, 2, 9-14.

Comisión Europea. (2012). La discriminación en la UE en 2012. Eurobarómetro. Retrieved from http://ec.europa.eu/commfrontoffice/publicopinion/index.cfm/ResultDoc/download/DocumentKy/57118

Comisión Europea. (2019). Discrimination in the European Union 2019. Eurobarómetro. Retrieved from https://ec.europa.eu/commfrontoffice/publicopinion/index.cfm/ResultDoc/download/DocumentKy/88338

Defensor del pueblo. (2000). Informe del Defensor del Pueblo. Violencia escolar: el maltrato entre iguales en la educación secundaria. Informes, estudios y documentos. doi:10.1007/ s13398-014-0173-7.2

Defensor del Pueblo. (2007). Informe del Defensor del Pueblo. Violencia escolar: el maltrato entre iguales en la Educación Secundaria Obligatoria 1999-2006. Informes, Estudios y Documentos. Retrieved from https:/www.defensordelpueblo.es/wp-content/ uploads/2015/05/2007-01-Violencia-escolar-el-maltrato-entre-iguales-en-la-EducaciónSecundaria-Obligatoria-1999-2006.pdf

Devís-Devís, J., Pereira-García, S., Valencia-Peris, A., Fuentes-Miguel, J., López-Cañada, E., \& Pérez-Samaniego, V. (2017). Harassment Patterns and Risk Profile in Spanish Trans Persons. Journal of Homosexuality, 64(2), 239-255. doi:10.1080/00918369.2016.1179027

Díaz, E. (2015). Acoso escolar, apoyo social y calidad de vida relacionada con la salud. (Tesis doctoral) Universidad de Castilla y La Mancha. Retrieved from https://ruidera.uclm.es/ xmlui/bitstream/handle/10578/8611/TESIS Díaz Herráiz.pdf?sequence=1\&isAllowed=y

Estévez, E., Murgui, S., Musitu, G., \& Moreno, D. (2008). Adolescent aggression: Effects of gender and family and school environments. Journal of Adolescence, 31(4), 433-450. doi:10.1016/j.adolescence.2007.09.007

Factor, R. J., \& Rothblum, E. D. (2007). A study of transgender adults and their nontransgender siblings on demographic characteristics, social support, and experiences of violence. Journal of LGBT Health Research, 3(3), 11-30. doi:10.1080/15574090802092879

Formby, E. (2013). The impact of homophobic and transphobic bullying on education and employment. Sheffield: Sheffield Hallam University. Retrieved from www4.shu.ac.uk/ research/ceir/sites/ceir/files/IGLYOBullyingReportJan2014.pdf

Garaigordobil, M., \& Oñederra, J. A. (2010). La violencia entre iguales Revisión teórica y estrategias de intervención (1a). Madrid: Pirámide.

García, F., \& Musitu, G. (2014). AF5. Autoconcepto Forma 5. Madrid, España: TEA.

Gómez, M. T., \& Navarro, D. (2017). Revisión teórica sobre bullying en alumnos con necesidades específicas de apoyo educativo. Psiquemag, 6(1). Retrieved from http:// ojs.ucvlima.edu.pe/index.php/psiquemag/article/view/169/88 
Grant, J. M., Mottet, L. a., Tanis, J., Herman, J. L., Harrison, J., \& Keisling, M. (2010). National transgender discrimination survey report on health and health care, 1-24. Retrieved from www.TransEquality.org\%5Cnwww.TheTaskForce.org

Grant, J. M., Mottet, L. a, Tanis, J., Harrison, J., Herman, J. L., \& Keisling, M. (2011). Injustice at Every Turn: A Report of the National Transgender Discrimination Survey. Washington National Center for Transgender Equality and National Gay and Lesbian Task Force, 25.

Greytak, E. A., Kosciw, J. G., \& Diaz, E. M. (2009). Harsh Realities: The experiences of transgender youth in our nation's school. Network, 67.

Grossman, A. H., \& D'Augelli, A. R. (2007). Transgender Youth and Life-Threatening Behaviors. Suicide and Life-Threatening Behavior, 37(5), 527-537. doi:10.1521/ suli.2007.37.5.527

Hill, D. B., \& Willoughby, B. L. B. (2005). The development and validation of the genderism and transphobia scale. Sex Roles, 53(7-8), 531-544. doi:10.1007/s11199-005-7140-x

Jones, T., \& Hillier, L. (2013). Comparing Trans-Spectrum and Same-sex-Attracted Youth in Australia: Increased Risks, Increased Activisms. Journal of LGBT Youth, 10(4), 287-307. doi:10.1080/19361653.2013.825197

Kosciw, Joseph G., Greytak, Emily A., Palmer, Neal A., \& Boesen, M. J. (2014). The 2013 National School Climate Survey. New York: GLSEN.

Kosciw, J. G., Greytak, E. A., Giga, N. M., Villenas, C., \& Danischewski, D. J. (2016). The 2015 National School Climate Survey. Retrieved from www.glsen.org

Lombardi, E. L., Wilchins, R. A., Priesing, D., \& Malouf, D. (2001). Gender Violence: Transgender Experiences with Violence and Discrimination. Journal of Homosexuality, 42(1), 89-101. doi:10.1300/J082v42n01

Magaz, A. M., Chorot, P., Santed, M. A., Valiente, R. M., \& Sandín, B. (2016). Evaluación del bullying como victimización: Estructura, fiabilidad y validez del Cuestionario de Acoso entre Iguales (CAI). Revista de Psicopatologia Y Psicologia Clinica, 21(2), 77-95. doi: 10.5944/rppc.vol.21.num.2.2016.16990

Platero, R. (Lucas). (2014). Trans*exualidades. Acompañamiento, factores de salud y recursos educativos (1a). Barcelona: Edicions Bellaterra.

Rigby, K., \& Slee, P. T. (1993). Dimensions of interpersonal relation among australian children and implications for psychological well-being. Journal of Social Psychology, 133(1), 33-42. doi:10.1080/00224545.1993.9712116

Russell, S. T., Ryan, C., Toomey, R. B., Diaz, R. M., \& Sanchez, J. (2011). Lesbian, Gay, Bisexual, and Transgender Adolescent School Victimization: Implications for Young Adult Health and Adjustment. Journal of School Health, 81(5), 223-230. doi:10.1111/ j.1746-1561.2011.00583.x

Serrano, A., \& Iborra, I. (2005). Violencia entre compañeros en la escuela. Centro Reina Sofía para el Estudio de la Violencia. España: Goaprint, S.L.

Shavelson, R. J., Hubner, J. J., \& Stanton, G. C. (1976). Self-Concept: Validation of Construct Interpretations. Review of Educational Research, 46(3), 407-441. https:// doi.org/10.3102/00346543046003407

Storch, E. A., \& Masia-Warner, C. (2004). The relationship of peer victimization to social anxiety and loneliness in adolescent females. Journal of Adolescence, 27(3), 351-362. doi:10.1016/j.adolescence.2004.03.003 
Vera, C., Vélez, C., \& García, H. (2017). Medición del bullying escolar: Inventario de instrumentos disponibles en idioma español. Revista Latinoamericana de Ciencia Psicológica, 1(1), 1-16. doi:10.5872/psiencia/9.1.31

Fecha de recepción: 23 de marzo de 2020.

Fecha de revisión: 23 de abril de 2020.

Fecha de aceptación: 14 de octubre de 2020. 